\title{
MAGNETIZATION, MATTER-ANTIMATTER SYMMETRY AND \\ THE BARYON-PHOTON RATIO IN THE UNIVERSE
}

\author{
M. A. MELVIN
}

Physics Dept., Temple University, Philadelphia, Pa. 19122, U.S.A.

\begin{abstract}
It is shown that the universal magnetic field, or sufficiently extended intergalactic fields, of magnitude $\gtrsim 10^{-9} \mathrm{G}$ would have aligned the magnetic moments of all leptons at an early time. Unless an upper limit to temperature exists, the alignment of all nucleons would also have occurred at an earlier time when the temperature was $m_{N} / m_{e} \sim 2000$ times higher. Possible inferences of this early magnetization of the matter in the universe for observation are discussed. The one selected for particular analysis is the parameter

$$
\frac{\text { No. baryons }}{\text { No. photons }}=\eta \approx 10^{-9}
$$

expressing the inverse 'hotness' of the universe. The matter-anti-matter symmetric theory of $\eta$ given by Omnes, amended by Steigman and Kundt is reviewed. The effect of the large scale magnetic alignment on the value of $\eta$ resulting from the annihilation era is then discussed by means of a model in which matter and antimatter droplets or filaments are in quasi-equilibrium under magnetization and effective surface forces. The magnetized droplets affect the diffusion of neutrons, which is dominant in the annihilation era.
\end{abstract}

